\title{
Harina de guayaba taiwanesa como sustituto de almidón en la producción de embutidos crudos y cocidos
}

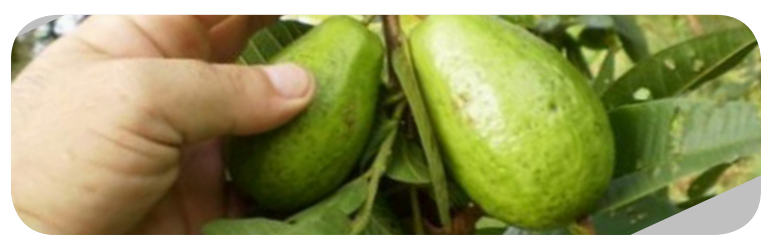

\author{
Walter Alexander Cosme Linares ${ }^{1}$ \\ Melba Jeannette Pacheco de Jordán ${ }^{2}$ \\ Blanca Estela Saravia Arias ${ }^{3}$ \\ Universidad Católica de El Salvador
}

Recepción: 21/04/2018 Aceptación: 17/06/2018

\section{Resumen}

La investigación se realizó en el laboratorio de investigación de la Universidad Católica de El Salvador sede Ilobasco, Cabañas en El Salvador, a raíz de aprovechar excedentes de producción de guayaba taiwanesa. De los excedentes de cosecha se recolectó y clasificó fruta para establecer calidad, protegiendo la inocuidad de los mismos; luego los frutos fueron rebanados sin sobrepasar $5 \mathrm{~mm}$ de ancho, ya que a mayor grosor menor deshidratación, inclusive a $60^{\circ} \mathrm{C}$ y velocidad del aire $3.5 \mathrm{~m} / \mathrm{s}$.

Luego de la obtención de harina de guayaba se determinaron los porcentajes a ser agregados a las diferentes fórmulas. Se evaluó y caracterizó la harina de guayaba mediante análisis proximal y análisis de textura; posterior a la caracterización se pulverizó y tamizó para luego experimentar con dos porcentajes de harina de guayaba $1.7 \%$ y $3.4 \%$ como sustituto de almidón en la elaboración de embutidos crudos y cocidos. Se puede afirmar por los resultados de los análisis organolépticos que es factible la elaboración de embutidos crudos y cocidos con harina de guayaba, ya que esta no altera ninguna de las cualidades organolépticas en la producción de embutidos crudos y cocidos.

Palabras clave: Harina de guayaba, embutido crudo y cocido, análisis organoléptico, análisis proximal.

\begin{abstract}
The research was conducted in the Research Laboratory of the Universidad Católica de El Salvador, Ilobasco, Cabañas, as a result of taking advantage of surplus production of Taiwanese guava. From the harvest surplus, the fruit was collected and classified to establish quality, protecting the safety of the same; then the fruits were sliced without exceeding $5 \mathrm{~mm}$ in width, since the thicker the less dehydrated, even at $60^{\circ} \mathrm{C}$ and the air speed $3.5 \mathrm{~m} / \mathrm{s}$.

After obtaining guava flour, the percentages to be added to the different formulas were determined. The flour was evaluated and characterized by proximal and texture analysis; after characterization, it was pulverized and sifted for experimenting two percentages of guava flour $1.7 \%$ and $3.4 \%$ as a substitute of scratch in the preparation of raw and cooked sausages. It can be affirmed by the results of the organoleptic analyses that it is feasible to make raw and cooked sausages with guava flour, since this does not affect any of the organoleptic qualities in their production.
\end{abstract}

Key words: Guava flour, raw and cooked sausage, organoleptic analysis, proximal analysis.

1. Maestro en Asesoría Educativa, docente investigador, Facultad Multidisciplinaria de Ilobasco, Cabañas; email: walter.cosme@catolica.edu.sv

2. Técnico en Ingeniería Química, docente investigadora, Facultad Multidisciplinaria de Ilobasco, Cabañas; email: melba.pacheco@catolica.edu.sv

3. Técnico en Lácteos y Cárnicos, docente investigadora, Facultad Multidisciplinaria de Ilobasco, Cabañas; email: blanca.saravia@catolica.edu.sv 


\section{Introducción}

La creación de un subproducto, en este caso harina a partir de fruto fresco de guayaba taiwanesa (Psydium guajava) variedad Taiwán 1 , representa un verdadero aprovechamiento de los excedentes de fruto no distribuido en el comercio formal o informal. Al mismo tiempo, con la harina de guayaba se buscó estudiar una de sus aplicaciones en la elaboración de productos cárnicos crudos y cocidos; para este caso de estudio se sometieron a pruebas organolépticas dos productos comercialmente conocidos como chorizo argentino y salchicha común, ambos con diferentes porcentajes de harina de guayaba $1.7 \%$ y $3.4 \%$.

Acorde al IV Censo Agropecuario 2007-2008 del Ministerio de Agricultura de El Salvador (MAG), la superficie sembrada de Guayaba representa unas $185 \mathrm{Mz}$, brindando con ello una cosecha de 51,293QQ. La guayaba de origen asiático ha sido difundida a nivel mundial, permitiendo un mejoramiento selectivo en las variedades; por ejemplo, la Variedad Taiwán 1 fue introducida en 1998 a El Salvador, por la Misión Internacional de Taiwán, posteriormente, en el año 2000, se introdujeron las variedades Taiwán 2, 3 y 4. Estas presentan características particulares en cuanto a rendimiento, nutrición y usos medicinales; destacando en mayor medida su gran tamaño y peso.

La corta vida útil de los frutos por razones naturales o microbiológicas está generalmente asociada con el hábitat de la fruta y la tempera- tura. En general, el tejido interno (mesocarpio) de los frutos se encuentra libre de carga microbiana, estando la flora asociada con la superficie externa conocida como cáscara, piel o epicarpio. Acorde a Barreiro y Sandoval (2006), se suman otros factores tales como: carencia de centros de acopio, mal estado de las vías rurales, vehículos no refrigerados e indebido almacenamiento; actividades inadecuadas de post cosecha y la falta de aplicación de Buenas Prácticas de Manufactura (BPM) hacen que mucha fruta se pierda durante el punto máximo de producción. Otros factores por los cuales se desperdicia la cosecha de guayaba son: bajo consumo, alta oferta estacionaria y precio poco accesible al consumidor final.

La alternativa propuesta a esta pérdida y desaprovechamiento es la industrialización encaminada a obtener productos que mantengan las características sensoriales y nutricionales de la guayaba, para así aumentar su consumo, aprovechar los excedentes de producción y obtener productos con valor agregado.

En El Salvador, la guayaba se consume en mayor cantidad como fruto fresco; en segundo plano de consumo, mediante néctares, mermeladas, jugos, almibares, dulces, helados y gelatinas, siendo estas las presentaciones de mayor manufactura en el país (Guía Técnica del Cultivo de la Guayaba. CENTA 2010). Sin embargo, el propósito de este estudio fue innovar con este insumo; por tal razón, se hicieron pruebas con harina de guayaba para ser agregada en sustitución del almidón, a la 
mezcla de ingredientes, en la elaboración de embutidos crudos y cocidos. Esto para aprovechar las propiedades de viscosidad, fibra y contenido de pectina que posee este fruto.

Uno de los métodos para conservar y diversificar la carne es mediante la producción de cárnicos. Estos surgen de una mezcla de carne (res, cerdo, pollo) picada, grasa, sales, condimentos y especias que contribuyen a potenciar el sabor del producto; y por último, embutido en tripas naturales o colágeno (Principios Básicos de Elaboración de Embutidos. Núm. 4/89 HD), da lugar a la incorporación de más ingredientes en la receta. La harina de guayaba no es algo nuevo, no obstante, es algo que no se había incorporado en las recetas de embutidos crudos y cocidos con anterioridad; siendo para este caso el chorizo argentino y la salchicha común.

\section{Materiales y Métodos}

El tipo de investigación fue experimental y aplicada, realizándose en tres fases: La primera consistió en la recolección-clasificación del fruto y el procesamiento de la harina; la segunda en la elaboración de chorizo tipo argentino y salchicha común; la tercera y última fase en los análisis organolépticos, proximal, microbiológicos y análisis de textura.

Cabe añadir que, durante la investigación se tuvo la colaboración de la finca San José, ubicada en el cantón Santa Cruz Porrillo, municipio de Tecoluca, departamento de San Vicente; así como a los alumnos del Técnico en Alimentos del Proyecto Becas y Estipendios del Ministerio de Educación, personal del Parque Tecnológico Agroindustrial, la Asociación de Desarrollo Comunal PLATANARES y el Ministerio de Educación de El Salvador.

Tabla 1. Clasificación de Guayaba según su estado de madurez

\begin{tabular}{|c|c|}
\hline Calidad de guayaba & Estado de madurez \\
\hline Primera & 110 días $^{4}$ \\
\hline Segunda & 112 días \\
\hline Tercera & 120 días \\
\hline
\end{tabular}

\begin{tabular}{|c|c|}
\hline Recolección de fruta & Calidad \\
\hline $1^{\circ}$ recolección & Segunda \\
\hline $2^{\circ}$ recolección & Segunda \\
\hline $3^{\circ}$ recolección & Tercera \\
\hline $4^{\circ}$ recolección & Tercera \\
\hline $5^{\circ}$ recolección & Segunda \\
\hline
\end{tabular}

Figura 1. Calidad del fruto para la producción de harina. 
Para la obtención de harina de guayaba se procedió a almacenar y seleccionar excedente de la cosecha de guayaba taiwanesa, tomando en cuenta la calidad basadas en sus tres estados de maduración. El proceso se explica a continuación por cada una de sus etapas:

a. Selección y clasificación de la fruta. Esto se realiza según su calidad. Posteriormente, se lavó y desinfectó en solución clorada a 50 partes por millón con Hipoclorito de Sodio (Manual para la preparación y venta de frutas y hortalizas, FAO) y se procedió a rebanar a $5 \mathrm{~mm}$ de grosor.

b. Deshidratado. El secado de la guayaba se realizó a una temperatura constante de $60^{\circ} \mathrm{C}$ y velocidad de aire $3.5 \mathrm{~m} / \mathrm{s}$. por 16 horas en horno solar.

c. Molienda. Una vez secadas las rebanadas de la guayaba se procedió a moler, empacando de inmediato lo triturado en bolsa de polipropileno para evitar contaminación y absorción de agua.

d. Tamizado. Este se realizó para descartar partículas de mayor tamaño y homogenizar en tamaño la harina; luego se empacó al vacío para evitar la absorción de humedad en el producto.

\section{Caracterización de la harina de guayaba}

a. Rendimiento (Fruta fresco-deshidratado). Para determinar el rendimiento del proceso de elaboración de la harina se empleó la siguiente fórmula:

Porcentaje de rendimiento $=$

$$
\frac{\text { Peso de harina obtenida (lb) }}{\text { Perso de materia prima ingresada (lb) }} \times 100
$$

\section{b. Elaboración de embutidos con harina} de guayaba. La elaboración de los embutidos crudos y cocidos se llevó a cabo en laboratorio de cárnicos de la UNICAES, en la Facultad Multidisciplinaria de Ilobasco, Cabañas. El tipo de producto cárnico crudo que se trabajó inicialmente fue chorizo argentino; y el producto cárnico cocido fue salchicha común. Para ello se utilizó carne de res, carne de cerdo, grasa dorsal de cerdo y carne mecánicamente deshuesada (CMD), verificando sus características fisicoquímicas de acuerdo a NSO 67.02.13:98; además de utilizar aditivos regulados en cuanto a su función y límites permitidos: fosfato, eritorbato, sal de cura y condimento (Códex Stan 192-1995). 
Tabla 2. Porcentaje de harina de guayaba en formulación de embutidos

\begin{tabular}{|c|c|}
\hline $\begin{array}{c}\text { Porcentaje de harina de guayaba en formu- } \\
\text { lación de embutido crudo chorizo argentino }\end{array}$ & $\begin{array}{c}\text { Porcentaje de harina de guayaba en formu- } \\
\text { lación de embutido cocido salchicha común }\end{array}$ \\
\hline $5 \%$ & $5 \%$ \\
\hline $4 \%$ & $4 \%$ \\
\hline $3.4 \%$ & $3.4 \%$ \\
\hline $2 \%$ & $2 \%$ \\
\hline $1.7 \%$ & $1.7 \%$ \\
\hline
\end{tabular}

Las razones de estos porcentajes fueron para evaluar el comportamiento de la harina en conjunto con el resto de la mezcla y así establecer un límite de uso de la harina en los productos cárnicos. El procedimiento de elaboración fue de acuerdo a los parámetros establecidos por Organización de las Naciones Unidas para la Alimentación y la Cultura (FAO).

Los ingredientes para la producción de chorizo argentino fueron mezclados de manera manual en el siguiente orden: mezcla de carne de res, carne mecánicamente deshuesada, grasa de cerdo, soya texturizada, harina de guayaba, sal de cura, eritorbato, sal yodada, fosfato, glutamato $y$, por último, los condimentos (pimienta negra, achiote, ajo en polvo, cebolla en polvo, cilantro, chile rojo, vinagre, agua y condimento chorizo). Finalmente se mezcló hasta obtener una pasta cárnica homogénea, dejándose reposar por 24 horas (esta etapa también se conoce como añejamiento, en la cual se desarrollan las reacciones de maduración de la pasta). Posteriormente, se procedió a embutir en tripa colágeno calibre 28 , amarrándose cada chorizo a una distancia de $8 \mathrm{~cm}$. Finalmente se empacó al vació y se almacenó a una temperatura de $4^{\circ} \mathrm{C}$ en refrigeración.

Por otro lado, los ingredientes para la elaboración de salchicha común fueron mezclados en el siguiente orden: carne de cerdo, carne mecánicamente deshuesada, grasa de cerdo, sal de cura, sal yodada, eritorbato, fosfato, azúcar, aislado de soya, harina de guayaba, harina de trigo, carragenina, condimento salchicha, cebolla en polvo, ajo en polvo, pimienta negra, nuez moscada y color carmín. Se mezcló hasta formar una emulsión cárnica, dejándose reposar por 24 horas a una temperatura de $4^{\circ} \mathrm{C}$. Posteriormente, se procedió a embutir en funda celulosa calibre 14, amarrándose cada salchicha a una distancia de $12 \mathrm{~cm}$. Se realizó el proceso de escaldado en agua a $80^{\circ} \mathrm{C}$ hasta que las salchichas alcanzaron una temperatura interna de $68^{\circ} \mathrm{C}$; sometiéndolas inmediatamente a un choque térmico con agua fría. Luego se procedió a empacar al vacío conservándolas en refrigeración a $4^{\circ} \mathrm{C}$. 
c. Formulaciones evaluadas. Para definir el mejor porcentaje de harina de guayaba en los productos embutidos crudos y cocidos se procedió a realizar pruebas fisicoquímicas en cada uno de ellos, como el análisis de humedad, análisis microbiológicos y sensoriales como olor, color, textura y sabor.

d. Análisis de humedad. Se realizó en una termobalanza, colocando un gramo de muestra de chorizo argentino en el platillo de la misma, a fin de distribuirlo uniformemente. Se bajó la tapa de la balanza para que la muestra iniciara la pérdida de humedad, tomando la lectura final transcurridos 20 minutos. El mismo procedimiento se realizó para la obtención de humedad en salchicha común (NMX-F-428-1982).

e. Análisis organoléptico. Se realizaron seis pruebas, las cuales fueron ejecutadas por estudiantes de segundo año de la carrera de Técnico en Lácteos y Cárnicos, que se imparte en la Facultad Multidisciplinaria de Ilobasco de la Universidad Católica de El Salvador en el departamento de Cabañas; y por cuatro catedráticos. Las pruebas sensoriales se realizaron en ambas formulaciones de embutido crudo y cocido con los diferentes porcentajes de harina de guayaba agregados $1.7 \%$ y $3.4 \%$. Las apreciacio- nes cualitativas de aceptación en cuanto a color, apariencia, olor, sabor y textura fueron expresadas en escala de uno a diez, siendo uno el nivel de desagrado y diez la excelencia del producto.

f. Análisis microbiológico. El análisis microbiológico se basó en la incubación de harina de guayaba a $35^{\circ} \mathrm{C}$ durante 48 horas en cajas PETRI FILM correspondiente a: Aeróbios mesófilos, Coliformes totales, Escherichia coli y Sthapylococcus aureus. Del mismo modo se procedió a analizar las muestras de embutido crudo y cocido con el fin de conocer la presencia o ausencia de microrganismo patógenos que incidían en la inocuidad de los productos.

g. Análisis proximal. El análisis proximal se realizó en el laboratorio de calidad de SARAM S.A. de C.V., ubicado en carretera hacia Sonsonate, $\mathrm{km} 27$ 1/2, municipio de Lourdes, departamento de La Libertad. Ahí se analizó la harina de guayaba, basados en la metodología AOAC 1995. Para establecer los contenidos básicos presentes tales como: proteína, vitaminas, grasa, humedad, entre otros.

h. Análisis de textura. El análisis de textura se llevó a cabo en el laboratorio de calidad de SARAM S.A. de C.V. para evaluar la porosidad y tamaño de las partículas presentes en la harina de guayaba. Se so- 
metió a siete diferentes tamices, siendo el tamiz de 30Mesh el de menor tamaño; y el tamiz de 8 Mesh el de mayor abertura de orificios.

\section{Resultados y Discusión}

Acorde a la revista colombiana Universitaria Científica (diciembre 2014), la harina de guayaba es uno de los alimentos funcionales que, al incluirla como ingrediente, brinda valor agregado, ya que contiene vitamina $\mathrm{C}$ y fibra. Esta, al estar con un porcentaje reducido de humedad, permite absorción de agua y grasa; por tal motivo, puede actuar como uno de los componentes en la formulación de embu- tidos crudos y cárnicos; así como extensor, permitiendo disminuir costos de producción en la preparación de los embutidos.

Durante las producciones se pudo observar que la harina de guayaba tiene capacidades ligantes. Gracias a la cantidad de pectina y fibra que posee permiten emulsionarse con el resto de los ingredientes, brindando una buena unión y logrando estabilidad en la presentación de los productos terminados (Ver tabla 3).

Se realizaron análisis proximal en las producciones de harina, los cuales son reportados en la tabla 4.

Tabla 3. Rendimiento de harina de guayaba

\begin{tabular}{|c|c|c|}
\hline Fruta fresca & Harina & Fibra gruesa \\
\hline 10 libras calidad 2 & 2.97 libras & 4.17 libras \\
\hline 15 libras calidad 2 & 4.85 libras & 6.25 libras \\
\hline 30 libras calidad 3 & 5.30 libras & 7.8 libras \\
\hline 50 libras calidad 3 & 8.83 libras & 13 libras \\
\hline 50 libras calidad 2 & 60 libras & 25 libras \\
\hline 50 libras calidad 2 & 60 libras & 25 libras \\
\hline 50 libras calidad 2 & 60 libras & 25 libras \\
\hline
\end{tabular}

Tabla 4. Análisis proximal - harina de guayaba, Finca San José

\begin{tabular}{|c|c|c|c|c|}
\hline Análisis & $\begin{array}{c}\text { Harina } \\
\text { Producción 1 }\end{array}$ & $\begin{array}{c}\text { Harina } \\
\text { Producción 2 }\end{array}$ & $\begin{array}{c}\text { Harina } \\
\text { Producción 3 }\end{array}$ & $\begin{array}{c}\text { Harina } \\
\text { Producción 4 }\end{array}$ \\
\hline Humedad & 7.51 & 7.68 & 9.56 & 7.20 \\
\hline Proteína & 6.61 & 6.56 & 5.64 & 6.70 \\
\hline Grasa & 0.78 & 0.60 & 0.60 & 0.56 \\
\hline Ceniza & 3.32 & 3.50 & 3.40 & 3.53 \\
\hline Calcio & 0.36 & 0.42 & 0.48 & 0.30 \\
\hline Fósforo & 0.40 & 0.43 & 0.48 & 0.39 \\
\hline
\end{tabular}

Fuente: SARAM S. A. de C. V. 
Tabla 5. Análisis de textura - harina de guayaba

\begin{tabular}{|c|c|c|c|c|}
\hline $\begin{array}{c}\text { Abertura } \\
\text { de Tamices }\end{array}$ & $\begin{array}{c}\text { Harina } \\
\text { Producción 1 }\end{array}$ & $\begin{array}{c}\text { Harina } \\
\text { Producción 2 }\end{array}$ & $\begin{array}{c}\text { Harina } \\
\text { Producción 3 }\end{array}$ & $\begin{array}{c}\text { Harina } \\
\text { Producción 4 }\end{array}$ \\
\hline T8 & $1.82 \%$ & $1.42 \%$ & $3.76 \%$ & $1.70 \%$ \\
\hline T10 & $0.72 \%$ & $1.23 \%$ & $2.75 \%$ & $0.85 \%$ \\
\hline T12 & $1.09 \%$ & $2.29 \%$ & $4.86 \%$ & $1.18 \%$ \\
\hline T16 & $14.96 \%$ & $14.16 \%$ & $16.56 \%$ & $14.87 \%$ \\
\hline T18 & $10.21 \%$ & $15.23 \%$ & $14.56 \%$ & $9.21 \%$ \\
\hline T20 & $11.31 \%$ & $13.14 \%$ & $11.50 \%$ & $11.41 \%$ \\
\hline T30 & $19.34 \%$ & $15.53 \%$ & $15.60 \%$ & $20.35 \%$ \\
\hline
\end{tabular}

Fuente. SARAM S. A. de C. V.

Análisis de chorizo argentino con harina de guayaba al $1.7 \%$

La vida de anaquel promedio de las producciones de embutido crudo fue de diez días en temperatura constante de $4^{\circ} \mathrm{C}$. No se pudo establecer correlación con la adicción de harina de guayaba al $1.7 \%$.

Se realizaron pruebas sensoriales al producto chorizo argentino con harina de guayaba al $1.7 \%$. Sin embargo, no fue percibida por los panelistas la incorporación de la misma en el embutido, respetando estándares de humedad por debajo del 30\%, según Reglamentos y Normas del Codex alimentarious.
Al producir un batch de chorizo argentino con el $1.7 \%$ de harina de guayaba, se observó que no cambió radicalmente en cuanto a color, sabor y textura. Se logró unificar la mezcla y homogenizar todos los ingredientes, además de la capacidad de retención del agua agregada, mostrando resultados aceptables (Ver tabla 6).

\section{Análisis de chorizo argentino al 3.4\%}

La vida de anaquel promedio de las producciones de embutido crudo fue de diez días en temperatura constante de $4^{\circ} \mathrm{C}$. No se pudo establecer correlación con la adicción de harina de guayaba al $3.4 \%$.

Tabla 6. Análisis microbiológico de chorizo argentino con harina de guayaba al 1.7\%

\begin{tabular}{|c|c|c|c|c|}
\hline Muestra & $\begin{array}{c}\text { Aeróbios } \\
\text { mesófilos }\end{array}$ & $\begin{array}{c}\text { Coliformes } \\
\text { totales }\end{array}$ & Escherichia coli & $\begin{array}{c}\text { Sthapylococcus } \\
\text { aureus }\end{array}$ \\
\hline Producción 1 & $13 \mathrm{UFC/g}$ & $0 \mathrm{UFC} / \mathrm{g}$ & $0 \mathrm{UFC} / \mathrm{g}$ & $2 \mathrm{UFC} / \mathrm{g}$ \\
\hline Producción 2 & $3 \mathrm{UFC/g}$ & $0 \mathrm{UFC} / \mathrm{g}$ & $0 \mathrm{UFC} / \mathrm{g}$ & $3 \mathrm{UFC} / \mathrm{g}$ \\
\hline Producción 3 & $106 \mathrm{UFC} / \mathrm{g}$ & $0 \mathrm{UFC} / \mathrm{g}$ & $0 \mathrm{UFC} / \mathrm{g}$ & $65 \mathrm{UFC} / \mathrm{g}$ \\
\hline Producción 4 & $2 \mathrm{UFC/g}$ & $0 \mathrm{UFC} / \mathrm{g}$ & $0 \mathrm{UFC} / \mathrm{g}$ & $2 \mathrm{UFC} / \mathrm{g}$ \\
\hline
\end{tabular}


Se determinó que el 3.4\% de harina de guayaba incorporado al chorizo argentino fue la cantidad adecuada, ya que esto no realizó ningún cambio en cuanto a color, sabor, textura y apariencia. Asimismo, se logró unificar la mezcla y homogenizar todos los ingredientes; además de la capacidad de retención del agua agregada, mostrando resultados aceptables (Ver tabla 7).

\section{Producción de Salchicha común al 1.7\%}

La vida de anaquel promedio de los embutidos fue de quince días en temperatura constante de $4^{\circ} \mathrm{C}$. Sin embargo, no se pudo establecer correlación con la adicción de harina de guayaba al 1.7\%. Al producir un batch de salchicha común con el $1.7 \%$ de harina de guayaba en su formulación no cambió radicalmente en cuanto a color, sabor y textura. Sin embargo, se logró unificar la mezcla y homogenizar todos los ingredientes; además de la capacidad de retención del agua agregada, pero mostrando resultados aceptables (Ver tabla 8).

\section{Resultados de Producción de Salchicha co- mún al 3.4\%}

La vida de anaquel promedio de los embutidos fue de quince días en temperatura constante de $4^{\circ} \mathrm{C}$. No se pudo establecer correlación con la adicción de harina de guayaba al 3.4\%. Al producir un batch de salchicha común con el $3.4 \%$ de harina de guayaba, se observó que fue la cantidad adecuada, ya que esto no realizó ningún cambio en cuanto a color, sabor y textura. Se logró unificar la mezcla y homogenizar todos los ingredientes, además de la capacidad de retención del agua agregada (Ver tabla 9 ).

En el análisis organoléptico se valoraron las variables de sabor, color, aroma y textura, con la colaboración de alumnos de segundo año del Técnico en Lácteos y Cárnicos de la Universidad Católica de El Salvador y la participación de docentes que imparten cátedra en el mismo técnico (Ver tabla 10).

Tabla 7. Análisis microbiológico de chorizo argentino con harina de guayaba al 3.4\%

\begin{tabular}{|c|c|c|c|c|}
\hline Muestra & $\begin{array}{c}\text { Aeróbios } \\
\text { mesófilos }\end{array}$ & $\begin{array}{c}\text { Coliformes } \\
\text { totales }\end{array}$ & Escherichia coli & $\begin{array}{c}\text { Sthapylococcus } \\
\text { aureus }\end{array}$ \\
\hline Producción 1 & $35 \mathrm{UFC} / \mathrm{g}$ & $0 \mathrm{UFC} / \mathrm{g}$ & $0 \mathrm{UFC} / \mathrm{g}$ & $4 \mathrm{UFC} / \mathrm{g}$ \\
\hline Producción 2 & $24 \mathrm{UFC/g}$ & $0 \mathrm{UFC} / \mathrm{g}$ & $0 \mathrm{UFC} / \mathrm{g}$ & $7 \mathrm{UFC} / \mathrm{g}$ \\
\hline Producción 3 & $105 \mathrm{UFC} / \mathrm{g}$ & $0 \mathrm{UFC} / \mathrm{g}$ & $0 \mathrm{UFC} / \mathrm{g}$ & $75 \mathrm{UFC} / \mathrm{g}$ \\
\hline Producción 4 & $7 \mathrm{UFC} / \mathrm{g}$ & $0 \mathrm{UFC} / \mathrm{g}$ & $0 \mathrm{UFC} / \mathrm{g}$ & $5 \mathrm{UFC} / \mathrm{g}$ \\
\hline
\end{tabular}


Tabla 8. Análisis microbiológico salchicha común con harina de guayaba al 1.7\%

\begin{tabular}{|c|c|c|c|c|}
\hline Muestra & $\begin{array}{c}\text { Aeróbios } \\
\text { mesófilos }\end{array}$ & $\begin{array}{c}\text { Coliformes } \\
\text { totales }\end{array}$ & Escherichia coli & $\begin{array}{c}\text { Sthapylococcus } \\
\text { aureus }\end{array}$ \\
\hline Producción 1 & $5 \mathrm{UFC} / \mathrm{g}$ & $0 \mathrm{UFC} / \mathrm{g}$ & $0 \mathrm{UFC} / \mathrm{g}$ & $2 \mathrm{UFC} / \mathrm{g}$ \\
\hline Producción 2 & $7 \mathrm{UFC} / \mathrm{g}$ & $0 \mathrm{UFC} / \mathrm{g}$ & $0 \mathrm{UFC} / \mathrm{g}$ & $1 \mathrm{UFC} / \mathrm{g}$ \\
\hline Producción 3 & $16 \mathrm{UFC} / \mathrm{g}$ & $0 \mathrm{UFC} / \mathrm{g}$ & $0 \mathrm{UFC} / \mathrm{g}$ & $1 \mathrm{UFC} / \mathrm{g}$ \\
\hline Producción 4 & $8 \mathrm{UFC} / \mathrm{g}$ & $0 \mathrm{UFC} / \mathrm{g}$ & $0 \mathrm{UFC} / \mathrm{g}$ & $0 \mathrm{UFC} / \mathrm{g}$ \\
\hline
\end{tabular}

Tabla 9. Análisis microbiológico salchicha con harina de guayaba al 3.4\%

\begin{tabular}{|c|c|c|c|c|}
\hline Muestra & $\begin{array}{c}\text { Aeróbios } \\
\text { mesófilos }\end{array}$ & $\begin{array}{c}\text { Coliformes } \\
\text { totales }\end{array}$ & Escherichia coli & $\begin{array}{c}\text { Sthapylococcus } \\
\text { aureus }\end{array}$ \\
\hline Producción 1 & $5 \mathrm{UFC} / \mathrm{g}$ & $0 \mathrm{UFC} / \mathrm{g}$ & $0 \mathrm{UFC} / \mathrm{g}$ & $1 \mathrm{UFC} / \mathrm{g}$ \\
\hline Producción 2 & $1 \mathrm{UFC} / \mathrm{g}$ & $0 \mathrm{UFC} / \mathrm{g}$ & $0 \mathrm{UFC} / \mathrm{g}$ & $2 \mathrm{UFC} / \mathrm{g}$ \\
\hline Producción 3 & $7 \mathrm{UFC} / \mathrm{g}$ & $0 \mathrm{UFC} / \mathrm{g}$ & $0 \mathrm{UFC} / \mathrm{g}$ & $2 \mathrm{UFC} / \mathrm{g}$ \\
\hline Producción 4 & $1 \mathrm{UFC} / \mathrm{g}$ & $0 \mathrm{UFC} / \mathrm{g}$ & $0 \mathrm{UFC} / \mathrm{g}$ & $0 \mathrm{UFC} / \mathrm{g}$ \\
\hline
\end{tabular}

Tabla 10. Análisis organoléptico embutido crudo y cocido

\begin{tabular}{|c|c|c|c|c|}
\hline Embutido crudo y cocido & Aroma & Color & Sabor & Textura \\
\hline Salchicha común $1.7 \%$ & 5.0 & 4.6 & 6.0 & 9.4 \\
\hline Salchicha común 3.4\% & 7.5 & 7.1 & 8.7 & 6.1 \\
\hline Chorizo argentino 1.7\% & 8.5 & 7.7 & 7.7 & 7.4 \\
\hline Chorizo argentino 3.4\% & 9.5 & 8.0 & 9.3 & 3.5 \\
\hline
\end{tabular}




\section{Conclusiones}

El estado de madurez de la guayaba destinada a la producción de harina debe ser en una etapa conocida coloquialmente como "sazona”; es decir, que aún no se haya alcanzado la madurez. Este punto será determinado por el tiempo y el cambio de tono en el color verde (Producción de Guayabas Taiwanesas, CENTA, 2002). Si la guayaba es procesada en un estado de maduración mayor, esta rendirá en menor proporción, ya que la testa posee mayor dureza y el potasio se ha concentrado en el fruto, brindándole a la guayaba una excelente dulzura para consumo en fresco, pero no para elaborar harina.

Durante esta etapa los azucares se caramelizan en el proceso de pulverización, representando un problema para el equipo utilizado (molinos). También el porcentaje de proteínas se ve afectado por el estado de madurez, ya que estas disminuyen. En cuanto a grasa, ceniza, calcio y fósforo, estos se mantienen al comparar los resultados.

En el análisis de humedad se observó que, a mayor estado de maduración, el porcentaje de humedad es superior que en otros. En promedio, la humedad en fruto sazón obtuvo un $7.44 \%$ y en fruto con mayor estado de maduración obtuvo un 9.56\%. Asimismo, se recomienda rebanar el fruto a $5 \mathrm{~mm}$ como grosor máximo, ya que, a mayor grosor, mayor número de horas para deshidratarse, elevando con ello los costos.
El crecimiento microbiológico en todos los productos: Escherichia coli, Coliformes totales, hongos y levaduras fue de 0UFC/g. En el caso de los análisis de Sthapylococcus aureus y Aerobios mesófilos sí hubo crecimiento; no obstante, este estuvo dentro de los parámetros aceptables. Es importante señalar que la tercera producción cuyo fruto tenía un estado de maduración mayor, presentó crecimiento mayor que las otras tres producciones. Por consiguiente, frutas con estado de madurez equivalente a daños irreversibles en ella, los cuales se detectan fácilmente por el cambio producido en una o más de sus características sensoriales; es decir, su apariencia, aroma, color, sabor y textura. No obstante, al someter el fruto al proceso de deshidratación por calor, el crecimiento microbiológico puede ser detenido y la contaminación sería a consecuencia del manejo post deshidratación.

El promedio de duración de vida útil de la harina de guayaba en condiciones de empaque y almacenamiento en bolsas de polipropileno sellado, térmicamente fue de 18 meses. En cuanto a la textura del fruto en estado de madurez, la harina de guayaba no puede ser tamizada en tamiz 30, únicamente en mayor envergadura. Esto es debido a las características y al comportamiento de los azucares, debido a la temperatura en el proceso de deshidratación y pulverización se carameliza, atrayendo así otras partículas las cuales forman grumos de mayor tamaño que no traspasan y obstruyen el tamiz. 
En los análisis microbiológicos obtenidos del proceso de fabricación de productos cárnicos crudos y cocidos, se obtuvo diferencias en los resultados. Sin embargo, no se puede establecer una correlación con la adición de harina de gayaba en ninguno de los porcentajes. Por naturaleza, en embutido crudo como el caso del chorizo argentino, se presentó en su mayoría crecimiento microbiológico de Aerobios mesófilos y Sthapylococcus aureus, los cuales están dentro de los parámetros establecidos por el codex alimentarius.

En cuanto a los embutidos cocidos, en el caso de salchichas comunes y sin hacer diferencia entre las formuladas con $1.7 \%$ de harina y las formuladas con $3.4 \%$ de harina como extensor, el crecimiento microbiológico fue mínimo. Esto a consecuencia del proceso de cocción al cual fue sometida la salchicha durante su procesamiento. Este detiene el crecimiento microbiológico, deduciéndose que cualquier contaminación pudo haber sido obtenida en la fase de post-producción.

Al comparar las dos formulaciones de salchicha se obtuvo mayor rendimiento, haciendo uso del 3.4\% de harina de guayaba, ya que permitió mayor absorción de líquidos y facilitó textura blanda que contribuyó a la compactación. Es importante reconocer que para este tipo de producciones el tamizado debe ser fino, pues por ser un producto cocido tiende a notarse las partículas gruesas de la harina; además de modificar la textura, siendo menos atractivo para los consumidores.

Ambas formulaciones resultaron eficientes en el procesamiento de embutidos crudos y cocidos; no obstante, se debe cumplir con los requerimientos técnicos y legales de cada producto. El 3.4\% del uso de harina de guayaba es un porcentaje idóneo, pues no afecta las características sensoriales de los productos, adaptándose satisfactoriamente a la formulación.

Cabe destacar que el uso de la harina debe provenir de frutos sazones, ya que esta forma tiende a adaptarse a los aditivos y condimentos que se mezclen. En formulaciones usadas con harina derivada de fruta con estado de madurez avanzado tiende a predominar olor y sabor a guayaba; lo que no es aceptable ni agradable en los embutidos, influyendo en una menor durabilidad del producto en anaquel. 


\section{Referencias}

Aleyda J., H. B. (20 de julio de 2016). Deshidratación de la Guayaba, una opción para su aprovechamiento integral. Recuperado de http://www.bdigital.unal.edu.co/8536/6/06_ Parte02_Cap04.pdf

Barreiro, J., Sandoval, A. (2006). Operaciones de Conservación de Alimentos por Bajas, Temperaturas. Equinoccio, Valle de Sartenejas, Baruta, Venezuela. ISSN: 768-328-455-6

Casaca, Á. D. (2016). El Cultivo de la Guayaba (Psidium guajava L.), 5. Recuperado de http:// www.dicta.hn/files/Guayaba,-2005.pdf

Consejo Nacional de Ciencia y Tecnología (2016). Recuperado de https://www.defensoria.gob. sv/images/stories/varios/NORMAS/EMBUTIDOS/nso67.02.13.98\%20EMBUTIDOS.pdf

Dergal, S. B. (2006). Química de los alimentos. México: Pearson Educación.

El Salvador, Viceministerio de Comercio e Industria del Ministerio de Economía 2007-2008. IV Censo Agropecuario

García., I. M. (2017). Guía Técnica del Cultivo de la Guayaba. Recuperado de http://www.centa. gob.sv/docs/guias/frutales/GUIA\%20CULTIVO\%20GUAYABA.pdf

García., I. M. (2017). Producción de Guayabas Taiwanesas. Recuperado de http://centa.gob.sv/ docs/guias/frutales/Guayaba.pdf

Garzón., O. (2016). Extensores en la Industria Cárnica. Recuperado de http://oliveiragarzon. blogspot.com/2009/11/extensores-en-la-industria-carnica.html

Gerber Products de México, S. A. de C. V.; Cámara Nacional de la Industria de Transformación y Departamento de Normas (1982). NMX-F428-1982. Alimentos. Determinación de Humedad. (Método Rápido de la Termobalanza). Recuperado de http://www.colpos.mx/ bancodenormas/nmexicanas/NMX-F-428-1982.PDF

Gutiérrez, G. S. D. (2015). Fisiología y Atributos de la Calidad de la Guayaba "pera" (Psidium guajava cv.) en Poscosecha. Red de REvistas Científicas de América Latina y el Caribe, España y Portugal. 
Helrich, K. (2017). AOAC: Official Methods of Analysis (1). Recuperado de https://law.resource. org/pub/us/cfr/ibr/002/aoac.methods.1.1990.pdf

Jiménez, H. B. (2016). Deshidratación de la Guayaba, una opción para su aprovechamiento integral. Recuperado de http://www.bdigital.unal.edu.co/8536/6/06_Parte02_Cap04.pdf

Marquina, V; Araujo, L; Ruíz, J; Rodríguez-Malaver A Vit P. (2008) Composición química y capacidad antioxidante en fruta, pulpa y mermelada de guayaba. Archivos Latinoamericanos de Nutrición; 58 (1), Caracas, Venezuela

McGuire, A. E. (2016). Evaluando Proteínas no Cárnicas para Mejorar Textura y Retención de Humedad. Recuperado de http://www.agromeat.com/42840/evaluando-proteinas-no-carnicas-para-mejorar-textura-y-retencion-de-humedad

México, Instituto Politécnico Nacional. (2006). Diseño y Desarrollo de Productos Alimenticios. UPDCE

Nicanor, A. B.; Dávila O., G y Ortíz M., A. (s.f.) Obtención de un aislado proteínico a partir de la semilla de guayaba (Psidium guajaba L.). Recuperado de http://www.smbb.com.mx/congresos\%20smbb/veracruz01/TRABAJOS/AREA_XIII/CXIII-74.pdf

SantaOlalla., F. J. (16 de mayo de 2016). Principios Básica de Elaboración de Embutidos. Recuperado de http://www.mapama.gob.es/ministerio/pags/biblioteca/hojas/hd_1989_04.pdf

Segura, L. P. (2017). Evaluación del comportamiento tecnológico de harina de guayaba (Psidium guajava L) como sustituto de la harina de trigo en un producto cárnico cocido tipo salchicha. Recuperado de http://repository.lasalle.edu.co/bitstream/handle/10185/21754/43121021_2017.pdf?sequence=1\&isAllowed $=\mathrm{y}$

Serpa, G. A. M. (2015). Comparación de dos técnicas de deshidratación de guayaba-pera (Psidium guajava L.) sobre los efectos del contenido de vitamina $\mathrm{C}$ y el comportamiento de las propiedades técnico-funcionales de la fibra dietaría. Revista Lasallista de Investigación,12 (1), 11-20; Corporación Universitaria Antioquia, Colombia. 